\title{
ABHANDLUNGEN UND BERICHTE AUS DEM STAATLICHEN MUSEUM FUR TIERKUNDE IN DRESDEN
}

\author{
Verantwortlicher Redakteur: Dr. RAINER EMMRICH \\ Redaktioneller Beirat: Dr. ALFRED FEILER - Dr. ROLF HERTEL - \\ Dipl.-Biol. RUDIGER KRAUSE - Dipl.-Biol. FRITZ JURGEN OBST
}

\section{Hinweise für unsere Autoren}

Die Publikationen des Staatlichen Museums für Tierkunde in Dresden dienen der Veröffentlichung von Arbeiten aus dem Museum sowie solchen, die ganz oder teilweise Material des Museums behandeln oder über Materialien berichten, von denen Belegstücke in die Sammlung des Museums kommen.

Die Arbeiten sollen eine Thematik aus den Wissensgebieten Taxonomie, Phylogenie, Evolution, Morphologie, Anatomie, Biologie, Okologie, Faunistik, Zoogeographie sowie den fachbezogenen Museumswissenschaften beinhalten. Arbeiten anderer Wissensgebiete sowie biographisch oder methodisch orientierte Arbeiten können aufgenommen werden, sofern sie zur Kenntnis der genannten Gebiete beitragen. Es werden nur Originalarbeiten veröffentlicht, die weder ganz noch teilweise bereits an anderer Stelle erschienen sind. Publikationen über Tiermaterial vom Gebiet der Deutschen Demokratischen Republik werden vorrangig behandelt.

Für Inhalt, sprachliche Gestaltung sowie Beachtung einer eventuellen Veröffentlichungsgenehmigung ihrer Arbeiten sind die Autoren selbst verantwortlich. Eine redaktionelle Bearbeitung der Manuskripte behält sich der Herausgeber vor. Die Autoren erhalten eine Eingangsbestätigung oder umfassendere Nachricht über die eingesandten Manuskripte. Nach Veröffentlichung ihrer Arbeiten erhalten die Autoren unentgeltlich 100 Sonderdrucke zugesandt; bei Veröffentlichungen, welche mehr als 200 Seiten umfassen, nur 50 Sonderdrucke. Von in den „Faunistischen Abhandlungen" erscheinenden Kurzmitteilungen werden 10 Belegexemplare ausgegeben. Diese Zahlen können aus technischen Gründen auch bei Veröffentlichungen von zwei oder mehr Autoren nicht erhöht werden.

Die Manuskripte können in deutscher, englischer oder französischer Sprache abgefaßt sein. Jede Arbeit (ausgenommen die für die „Reichenbachia“ vorgesehenen Veröffentlichungen) muß eine kurze Zusammenfassung in der zur Abfassung des Manuskriptes verwendeten Sprache enthalten. Diese Zusammenfassung wird einschließlich des Titels der Arbeit bei deutschsprachigen Manuskripten in das Englische, bei fremdsprachigen Manuskripten in das Deutsche übertragen. Es ist erwünscht, daß die Autoren diese Obertragung selbst vornehmen.

Eine Rezension eingesandter Monographien wird auf Wunsch gern vorgenommen.

Spezielle Richtlinien über Manuskriptgestaltung, Ausführung der Illustrationen und Korrekturgang der Publikationen können auf Anforderung vom Herausgeber bezogen werden. 